University of Wollongong

Research Online

Australian Institute for Innovative Materials -

Papers

Australian Institute for Innovative Materials

$1-1-2018$

\title{
Hierarchical Porous NiO/b-NiMoO4 Heterostructure as Superior Anode Material for Lithium Storage
}

Zhijian Wang

Hubei University

Shilin Zhang

University of Wollongong, sz384@uowmail.edu.au

Hai Zeng

South-Central University for Nationalities

Haimin Zhao

Tianneng Battery Group Co Ltd

Wei Sun

Tianneng Battery Group Co Ltd

See next page for additional authors

Follow this and additional works at: https://ro.uow.edu.au/aiimpapers

Part of the Engineering Commons, and the Physical Sciences and Mathematics Commons

Research Online is the open access institutional repository for the University of Wollongong. For further information contact the UOW Library: research-pubs@uow.edu.au 


\title{
Hierarchical Porous NiO/b-NiMoO4 Heterostructure as Superior Anode Material for Lithium Storage
}

\author{
Abstract \\ Ternary transition metal oxides (TTMOs) have attracted considerable attention for rechargeable batteries \\ because of their fascinating properties. However, the unsatisfactory electrochemical performance \\ originating from the poor intrinsic electronic conductivity and inferior structural stability impedes their \\ practical applications. Here, the novel hierarchical porous NiO/ $\beta$-NiMoO4heterostructure is fabricated, \\ and exhibits high reversible capacity, superior rate capability, and excellent cycling stability in Li-ion \\ batteries (LIBs), which is much better than the corresponding single-phase NiMoO4and NiO materials. \\ The significantly enhanced electrochemical properties can be attributed to its superior structural \\ characteristics, including the large surface area, abundant pores, fast charge transfer, and catalytic effect \\ of the intermediate product of metallic nickel. The $\mathrm{NiO} / \beta$-NiMoO4heterostructure delivers a high capacity \\ of $1314 \mathrm{mAhg}-1$ at $0.2 \mathrm{Ag}-1$ after 100 cycles. Furthermore, even after 400 cycles at $1 \mathrm{Ag}-1$, the reversible \\ capacity remains at around $500 \mathrm{mAhg}-1$. These results indicate that the $\mathrm{NiO} / \mathrm{B}$-NiMoO4heterostructure \\ shows great potential as an anode material for high-performance LIBs.
}

\section{Keywords}

superior, heterostructure, nio/b-nimoo4, porous, lithium, hierarchical, material, anode, storage

\section{Disciplines}

Engineering | Physical Sciences and Mathematics

\section{Publication Details}

Wang, Z., Zhang, S., Zeng, H., Zhao, H., Sun, W., Jiang, M., Feng, C., Liu, J., Zhou, T., Zheng, Y. \& Guo, Z. (2018). Hierarchical Porous NiO/b-NiMoO4 Heterostructure as Superior Anode Material for Lithium Storage. ChemPlusChem, 83 (10), 915-923.

\section{Authors}

Zhijian Wang, Shilin Zhang, Hai Zeng, Haimin Zhao, Wei Sun, Meng Jiang, Chuanqi Feng, Jianwen Liu, Tengfei Zhou, Yang Zheng, and Zaiping Guo 


\title{
Hiencie Hierarchical Porous $\mathrm{NiO} / \boldsymbol{\beta}-\mathrm{NiMoO}_{4}$ Heterostructure as Superior Anode Material for Lithium Storage
}

\author{
Zhijian Wang ${ }^{+[a]}$ Shilin Zhang, ${ }^{+\left[{ }^{[b]}\right.}$ Hai Zeng, ${ }^{[c]}$ Haimin Zhao, ${ }^{[\mathrm{d}]}$ Wei Sun, ${ }^{[\mathrm{d}]}$ Meng Jiang, ${ }^{[a]}$ \\ Chuanqi Feng, ${ }^{[a]}$ Jianwen Liu, ${ }^{[a]}$ Tengfei Zhou, ${ }^{[b, c, e]}$ Yang Zheng, ${ }^{*[b]}$ and Zaiping Guo*[a,b]
}

\begin{abstract}
Ternary transition metal oxides (TTMOs) have attracted considerable attention for rechargeable batteries because of their fascinating properties. However, the unsatisfactory electrochemical performance originating from the poor intrinsic electronic conductivity and inferior structural stability impedes their practical applications. Here, the novel hierarchical porous $\mathrm{NiO} / \beta$ $\mathrm{NiMoO}_{4}$ heterostructure is fabricated, and exhibits high reversible capacity, superior rate capability, and excellent cycling stability in Li-ion batteries (LIBs), which is much better than the corresponding single-phase $\mathrm{NiMoO}_{4}$ and $\mathrm{NiO}$ materials. The significantly enhanced electrochemical properties can be attributed to its superior structural characteristics, including the large surface area, abundant pores, fast charge transfer, and catalytic effect of the intermediate product of metallic nickel. The $\mathrm{NiO} / 3-\mathrm{NiMoO}_{4}$ heterostructure delivers a high capacity of $1314 \mathrm{~mA} \mathrm{hg}^{-1}$ at $0.2 \mathrm{Ag}^{-1}$ after 100 cycles. Furthermore, even after 400 cycles at $1 \mathrm{Ag}^{-1}$, the reversible capacity remains at around $500 \mathrm{mAhg}^{-1}$. These results indicate that the $\mathrm{NiO} / \beta$ $\mathrm{NiMoO}_{4}$ heterostructure shows great potential as an anode material for high-performance LIBs.
\end{abstract}

\section{Introduction}

High-performance rechargeable Li-ion batteries (LIBs) are gradually becoming indispensable units in portable commercialized electronics, electric vehicles, and renewable energy storage systems. ${ }^{[1,2]}$ Nevertheless, the conventional LIBs based on the prevailing carbon-based intercalation materials are already reaching their energy capacity limits. Hence, tremendous efforts have been devoted to exploring novel anode materials with higher energy densities, sustainable lifespans, cost-effec- tiveness, and safe working potentials, to make these materials applicable for the advanced LIBs required to meet the evergrowing demands for electrical applications. ${ }^{[3-5]}$

Ternary transition metal oxides (TTMOs) are emerging as promising candidate electrode materials for high-energy LIBs owing to their distinctive properties. ${ }^{[6,7]}$ It has been widely demonstrated that TTMOs generally exhibit superior reversible capacity, good structural stability, and high electronic conductivity. Specifically, the synergetic effects associated with their complex chemical compositions typically contribute to remarkable electrochemical activities and high specific capacities, and the multiple valences of the cations are beneficial for achieving more versatile redox reactions. ${ }^{[8,9]}$ More importantly, because of the lower activation energy for electron transfer between different cations, they normally possess higher electrical conductivities than their corresponding binary metal oxide counterparts. ${ }^{[10]}$ Among various potential TTMOs, spinel-structure nickel molybdate $\left(\mathrm{NiMoO}_{4}\right)$ has been regarded as an attractive material in view of its high theoretical capacity (about $984 \mathrm{mAhg}^{-1}$ ), good electrical conductivity, environmental friendliness, and low cost. ${ }^{[11,12]}$ At atmospheric pressure, there are two phases of $\mathrm{NiMoO}_{4}$ both with a monoclinic structure: $\alpha$ $\mathrm{NiMoO}_{4}$ (low-temperature stable phase) and $\beta-\mathrm{NiMoO}_{4}$ (hightemperature stable phase). The most striking difference between those two polymorphs is the coordination of the $\mathrm{Mo}^{6+}$ ions, with pseudo-octahedral coordination in the $\alpha-\mathrm{NiMoO}_{4}$ phase and distorted tetrahedral coordination in the $\beta-\mathrm{NiMoO}_{4}$ phase. ${ }^{[13]}$ Generally, $\beta-\mathrm{NiMoO}_{4}$ has a higher intrinsic conductivity and reactivity than $\alpha-\mathrm{NiMoO}_{4}{ }^{[14]}$ but it is very difficult to prepare and maintain. In addition, $\mathrm{NiMoO}_{4}$ electrode materials suffer from some drawbacks such as the huge volume change and low electrochemical reversibility, leading to inferior elec-

[a] Z. Wang, ${ }^{+}$M. Jiang, Prof. Dr. C. Feng, Dr. J. Liu, Prof. Dr. Z. Guo Hubei Collaborative Innovation Centre for Advanced Organic Chemical Materials Ministry of Education Key Laboratory for Synthesis and Applications of Organic Functional Molecules Hubei University, Wuhan 430062 (P. R. China)

[b] S. Zhang, ${ }^{+}$Dr. T. Zhou, Dr. Y. Zheng, Prof. Dr. Z. Guo Institute for Superconducting and Electronic Materials School of Mechanical, Materials, and Mechatronics Engineering University of Wollongong

North Wollongong, NSW 2500 (Australia) E-mail:yz966@uowmail.edu.au zguo@uow.edu.au

[c] H. Zeng, Dr. T. Zhou

Key Laboratory of Catalysis and Materials Science of the State Ethnic Affairs Commission \& Ministry of Education

South-Central University for Nationalities

Wuhan, 430074 (P. R. China)

[d] H. Zhao, W. Sun

Tianneng Battery Group Co. Ltd

18 Baoqiao Road, Huaxi Industrial Functional Zone Changxing

Zhejiang, 313100 (P. R. China)

[e] Dr. T. Zhou

Key Laboratory of Advanced Energy Materials Chemistry (Ministry of Education), Nankai University Tianjin 300071 (P. R. China)

$\left.{ }^{+}\right]$These authors contributed equally to this work.

$\square$ Supporting information and the ORCID identification number(s) for the

(iD author(s) of this article can be found under: https://doi.org/10.1002/cplu.201800220.

तÛứ, This article is part of a Special Issue on "Future Energy Technology". To view the complete issue, visit: https://doi.org/10.1002/cplu.v83.10. 
trochemical performance. ${ }^{[15,16]}$ Therefore, the rational design and facile synthesis of $\beta-\mathrm{NiMoO}_{4}$ materials for high-energy LIBS remains a significant challenge.

Constructing heterostructures by combining active materials with metal oxides or sulfides has proven to be an effective way to conquer the abovementioned obstacles. ${ }^{[17,18]}$ It is believed that such constructed heterostructures usually enable abundant electrochemical active sites, short ion diffusion paths, and good structural stability originating from the synergistic effect of different components, and therefore exhibit better electrochemical performances. ${ }^{[19]}$ For example, Yuan et al. developed hierarchical mesoporous $\mathrm{ZnO} / \mathrm{ZnFe}_{2} \mathrm{O}_{4}$ sub-microcubes, which exhibited better electrochemical Li-storage properties than the single-phase $\mathrm{ZnFe}_{2} \mathrm{O}_{4}$, owing to the striking synergistic effect between the bicomponent active, well-dispersed $\mathrm{ZnO}$ and ZFO phases at the nanoscale. ${ }^{[20]}$ Mai et al. found that hierarchical $\mathrm{MnMoO}_{4} / \mathrm{CoMoO}_{4}$ heterostructured nanowires showed enhanced specific capacitance and reversibility compared with the $\mathrm{MnMoO}_{4}$ and $\mathrm{CoMoO}_{4}$ electrodes. ${ }^{[21]}$ Similarly, other hybrid heterostructures such as $\mathrm{ZnCo}_{2} \mathrm{O}_{4} / \mathrm{NiO}$ core/shell nanowires, ${ }^{[22]} \mathrm{NiO}-\mathrm{Co}_{3} \mathrm{O}_{4}$ nanoplates, ${ }^{[23]} \mathrm{CoO} / \mathrm{CoFe}_{2} \mathrm{O}_{4}$ nanocomposites, ${ }^{[24]}$ and $\mathrm{CoMoO}_{4} / \mathrm{Co}_{3} \mathrm{O}_{4}$ composites ${ }^{[25]}$ have been employed as LIB electrodes and demonstrated improved reversible capacity and cycling performance.

Besides the favorable components, the structure also plays an important role in determining the electrochemical properties of the developed electrode materials. It is considered that the hierarchical architectures constructed from 2D building blocks possess superior structural characteristics with large specific surface areas, more accessible pores, and high structural stabilities, making them promising candidates for energy-related applications. ${ }^{[26]}$ Undoubtedly, the large specific surface would provide rich Li-storage active sites, and the generated numerous pores may offer additional space to accommodate the volume expansion, consequently maintaining the structural stability and electrode integrity. Lou et al. synthesized the hierarchical tubular structure of Co-Mn mixed metal oxides, manifesting excellent electrochemical properties upon employment as electrodes in Li-ion batteries and hybrid supercapacitors. ${ }^{[27]}$ A hierarchical fascicular structure of $\mathrm{Zn}_{2} \mathrm{GeO}_{4}$ anode obtained through a wet chemistry method also exhibited remarkable Liion storage performance, as the hierarchical structure could alleviate the volume changes and retain the whole configuration, and the small building units enlarged the electrode/electrolyte contact area and constructed an integrated interlaced conductive network. ${ }^{[28]}$

Herein, we have designed and constructed a novel hierarchical porous $\mathrm{NiO} / \beta-\mathrm{NiMoO}_{4}(\mathrm{NNMO})$ heterostructure through a facile hydrothermal technique followed by a calcination process. Interestingly, the NiO phases in the NNMO heterostructure could be regarded as a multifunctional layer, which can not only contribute to the high theoretical capacity, but also function as a mechanical buffer to alleviate the huge volume changes in the $\mathrm{NiMoO}_{4}$ matrix, thus offering high capacity and good structural stability. Furthermore, the generated metallic nickel (Ni) nanoparticles from the lithiation reaction of $\mathrm{NiO}$ nanosheets with $\mathrm{Li}$ are able to promote the reversible decom- position of $\mathrm{Li}_{2} \mathrm{O}$ during the delithiation process, leading to a high initial Coulombic efficiency and enhanced reversible capacity of the $\mathrm{NiMoO}_{4}$. In addition, the abundant pores produced from the surface interconnected nanosheets and the self-assembled microspheres with hierarchical structure provide more space to relieve the induced stress during the lithiation/delithiation processes and preserve the structural integrity. As expected, the as-prepared $\mathrm{NiO} / \beta-\mathrm{NiMoO}_{4}$ heterostructured anode exhibited much better electrochemical Li storage properties than its single-phase $\mathrm{NiO}$ or $\mathrm{NiMoO}_{4}$ counterparts. Such a significantly enhanced electrochemical performance can be attributed to its superior structural characteristics achieved by rational structure engineering of the electrode.

\section{Results and Discussion}

The synthesis of the hierarchical porous $\mathrm{NiO} / \beta-\mathrm{NiMoO}_{4}$ (NNMO) heterostructure through a novel and facile strategy is illustrated in Scheme 1. First, appropriate proportions of $\mathrm{Ni}^{2+}$ and $\mathrm{MoO}_{4}{ }^{2-}$ precursors were mixed homogeneously into deionized water under magnetic stirring for a certain time, so that the acetate could be adsorbed on the surface of $\mathrm{MoO}_{4}{ }^{2-}$ by electrostatic interaction, and subsequently, the NNMO was obtained by oxidation of the as-prepared precursor mixture at $550^{\circ} \mathrm{C}$ under an oxygen atmosphere. For further comparison, single-phase $\mathrm{NiO}(\mathrm{NO})$ and $\mathrm{NiMoO}_{4}(\mathrm{NMO})$ were also fabricated through similar processes, but with different precursors chosen.

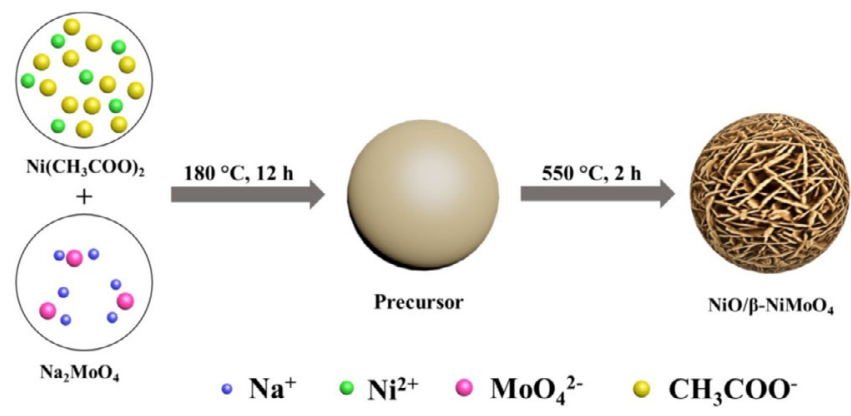

Scheme 1. Schematic illustration of the novel hierarchical porous $\mathrm{NiO} / \beta-\mathrm{Ni}$ $\mathrm{MoO}_{4}$ (NNMO) heterostructure prepared through a facile hydrothermal method followed by a calcination process.

The purity and crystal structure of the fabricated samples were analyzed by powder X-ray diffraction (XRD). The XRD patterns in Figure 1 a confirm that the as-prepared NNMO materials are composed of pure $\beta$-phase $\mathrm{NiMoO}_{4}$ (JCPDS Card No. 120348) and cubic bunsenite NiO (JCPDS Card No. 47-1049), indicating the coexistence of pure $\beta-\mathrm{NiMoO}_{4}$ and $\mathrm{NiO}$ phases in the obtained NNMO composite. Furthermore, the formation of the cubic NiO phase in the hybrid heterostructure is revealed by the diffraction peaks centered at $37.25^{\circ}, 43.28^{\circ}$, and $62.89^{\circ}$, corresponding to the (111), (200), and (220) crystal planes, respectively. The remarkable diffraction peak at the value of $26.65^{\circ}$ can be indexed to the characteristic (220) planes of $\beta$ $\mathrm{NiMoO}_{4}$, suggesting the high purity of the $\beta$-phase $\mathrm{NiMoO}_{4}$, 

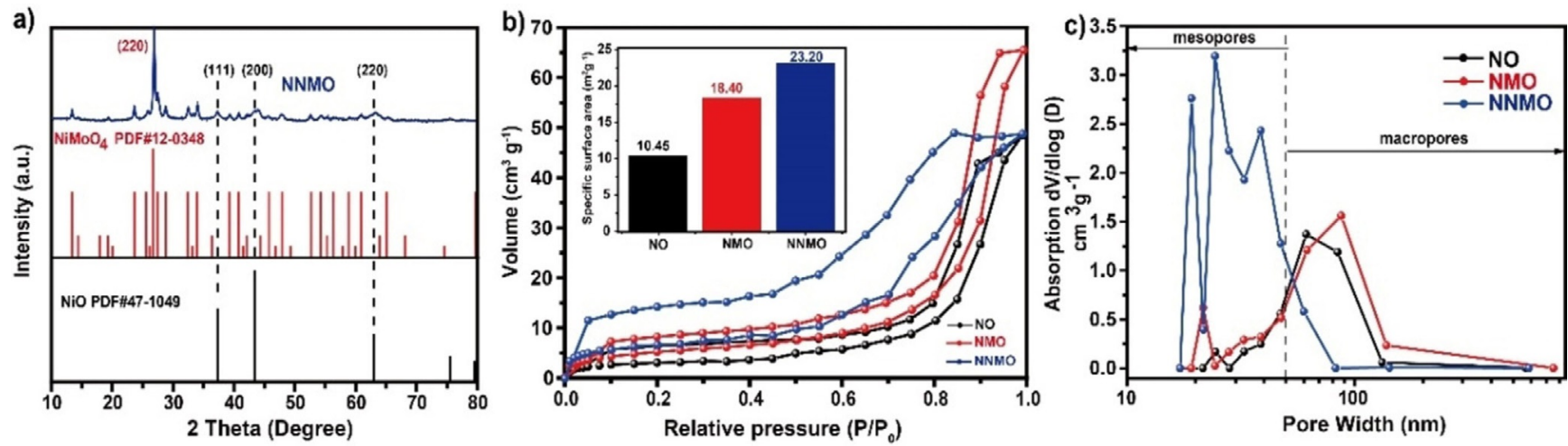

Figure 1. a) General XRD pattern of as-prepared NNMO; b) $\mathrm{N}_{2}$ adsorption/desorption isotherms (inset: comparison of the specific surface areas of NO, NMO, and NNMO); and c) pore-size distributions of the three samples.

without any trace/residual of the $\alpha$-phase. ${ }^{[29]}$ As shown in Figure S1 a (Supporting Information), the diffraction patterns of the prepared single-phase NO are well matched with those of pure NiO (JCPDS Card No. 47-1049), whereas all the diffraction peaks in Figure S1 b (Supporting Information) can be assigned to the pure monoclinic $\alpha-\mathrm{NiMoO}_{4}$ (JCPDS Card No. 33-0948). Notably, no diffraction peaks can be indexed to $\beta$-phase $\mathrm{NiMoO}_{4}$

$\mathrm{N}_{2}$ adsorption/desorption measurements were performed to analyze the physical properties of the obtained samples (Figure $1 \mathrm{~b}, \mathrm{c}$ ). As presented in Figure $1 \mathrm{~b}$, the isotherm curves of all three samples show distinct type IV adsorption isotherms with $\mathrm{H} 1$-type hysteresis loops within a relative pressure $P / P_{0}$ range from 0.05 to 0.95 in the desorption isotherm, implying the existence of numerous pores. On the basis of the BET method analysis, it was found that the NNMO has a much larger specific surface area of $23.20 \mathrm{~m}^{2} \mathrm{~g}^{-1}$, with a total pore volume of $2.4 \mathrm{~cm}^{3} \mathrm{~g}^{-1}$, compared with the single-phase NO $\left(10.45 \mathrm{~m}^{2} \mathrm{~g}^{-1}\right)$ and NMO $\left(18.40 \mathrm{~m}^{2} \mathrm{~g}^{-1}\right.$ ) (inset in Figure $1 \mathrm{~b}$ ). Accordingly, the BJH pore-size distribution profiles derived from the adsorption branches of the isotherms were also calculated and are shown in Figure $1 \mathrm{c}$. The pore-size distribution curves for NNMO reveal that most of the pores range between 20 and $50 \mathrm{~nm}$, markedly confirming the existence of a kind of mesoporous nanostructure. In addition, the narrow pore-size distributions of NO and NMO show that the numerous pores are mainly macropores. Impressively, the unique structural features, including the high specific surface area and abundant porous structure of the NNMO heterostructure, will not only provide many electroactive sites, a large electrode/electrolyte contact area, and easy electrolyte access, but will also accommodate the huge volume changes of the electrode during repeated lithiation/delithiation processes, ${ }^{[8,30,31]}$ thus making it extremely beneficial for high-rate performance and long cycle stability for Li-ion storage.

The chemical states and elemental composition of the assynthesized sample were further characterized by X-ray photoelectron spectroscopy (XPS). As displayed in Figure $2 \mathrm{a}$, the whole survey scan of the NNMO primarily demonstrates the presence of the elements $\mathrm{Ni}, \mathrm{Mo}$, and $\mathrm{O}$. The high-resolution spectrum of $\mathrm{Ni} 2 \mathrm{p}$ (Figure $2 \mathrm{~b}$ ) can be deconvoluted into two main peaks by using the Gaussian fitting method, with the binding energies centered at 855.9 and $873.8 \mathrm{eV}$, corresponding to two spin-orbit doublets $\left(\mathrm{Ni}_{2} \mathrm{p}_{3 / 2}\right.$ and $\mathrm{Ni} 2 \mathrm{p}_{1 / 2}$ ) and two weak shake-up satellite peaks of nickel located at 861.5 and $880.1 \mathrm{eV}$, respectively. ${ }^{[32,33]}$ The spin-energy separation of $17.9 \mathrm{eV}$ is characteristic of the presence of $\mathrm{Ni}^{2+} \cdot{ }^{[34]}$ On the

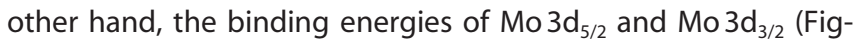
ure 2 c) are located at 232.0 and $235.1 \mathrm{eV}$, respectively. The binding energies and energy separation $(3.1 \mathrm{eV})$ are characteristics of the $\mathrm{Mo}^{6+}$ oxidation state in $\mathrm{NiMoO}_{4}{ }^{[35]}$ Figure $2 \mathrm{~d}$ clearly shows the high-resolution spectrum in the $\mathrm{O} 1 \mathrm{~s}$ region, which consists of a main peak located at $531.7 \mathrm{eV}$ coupled with a shoulder at $532.7 \mathrm{eV}$. Therefore, the XPS results indicate the presence of both $\beta-\mathrm{NiMoO}_{4}$ and $\mathrm{NiO}$ in the composite, which is consistent with the XRD result (Figure $1 \mathrm{a}$ ).

The detailed morphology and microstructure of the as-prepared hierarchical porous $\mathrm{NiO} / \beta-\mathrm{NiMoO}_{4}$ heterostructure were investigated by scanning electron microscopy (SEM) and highresolution transmission electron microscopy (HRTEM). Figure $3 \mathrm{a}$ and $\mathrm{b}$ show representative SEM images of the NNMO at low and high magnification, respectively. It can be seen that the NNMO shows typical hierarchical porous microspheres, constructed from a large number of nanosheets with a thickness of $10-20 \mathrm{~nm}$. Moreover, close observation reveals that there are numerous nanopores generated on the nanosheets (Figure $3 \mathrm{~b}$ ) and the assembled nanosheets also form abundant large pores, which is also consistent with the BET results. The TEM images further confirm the hierarchical porous structure of the NNMO sample (Figure $3 c, d$ ) The pores could promote electrolyte access to the interior portion of the material and offer enough additional space to accommodate the volume changes of the electrodes, thus improving electrochemical performance for Li-ion storage. Furthermore, the marked interplanar distances in the high-resolution TEM image are 0.336 and $0.241 \mathrm{~nm}$ (Figure $3 \mathrm{e}$ ), corresponding to the (220) and (111) lattice planes of $\beta-\mathrm{NiMoO}_{4}$ and $\mathrm{NiO}$, respectively, certifying the presence of the two phases in the composite. More interestingly, the $\beta-\mathrm{NiMoO}_{4}$ and $\mathrm{NiO}$ nanosheets are in tight contact with each other and form the heterostructure, which may promote rapid ion/electron transfer. ${ }^{[18]}$ In addition, the introduced $\mathrm{NiO}$ phase could function as the buffer layer to accommodate 
a)
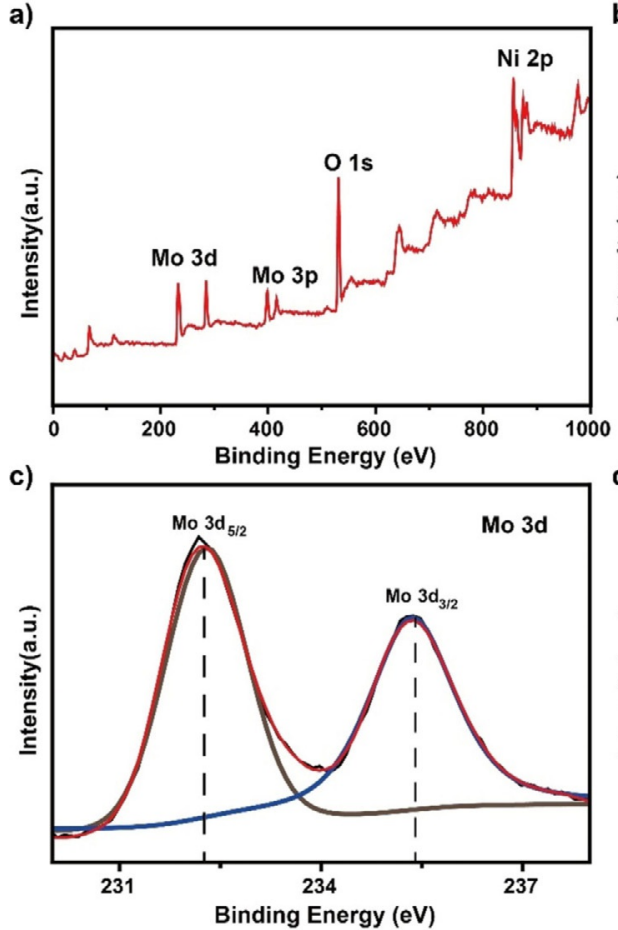

b)
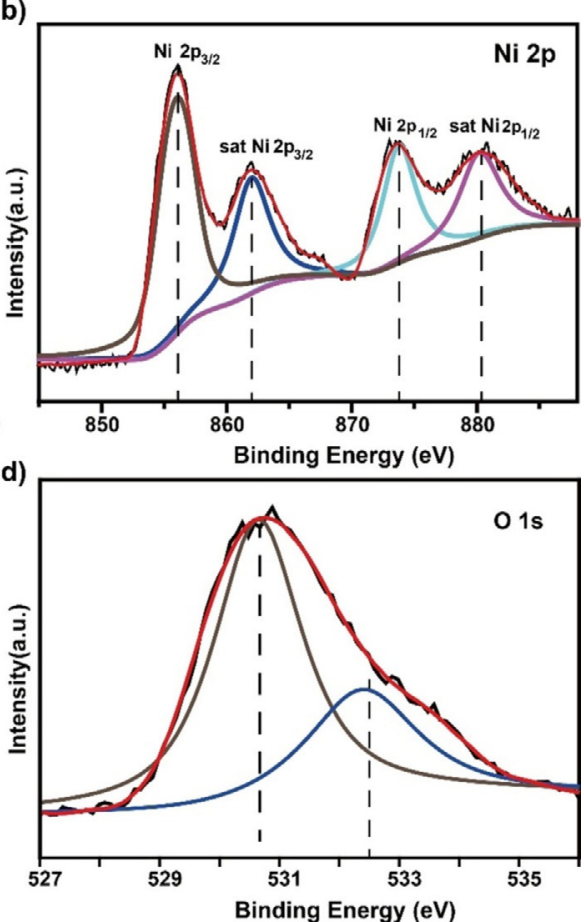

Figure 2. a) XPS survey spectrum, b) high-resolution Ni 2p spectrum, c) Mo 3d spectrum, and d) O 1s region of the obtained NNMO material.
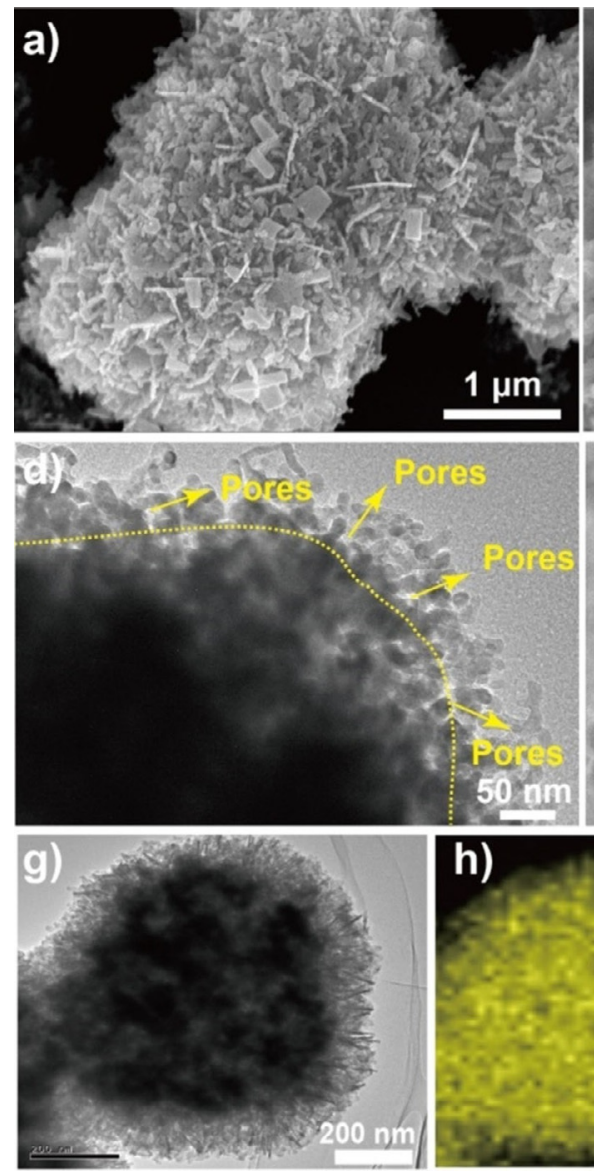
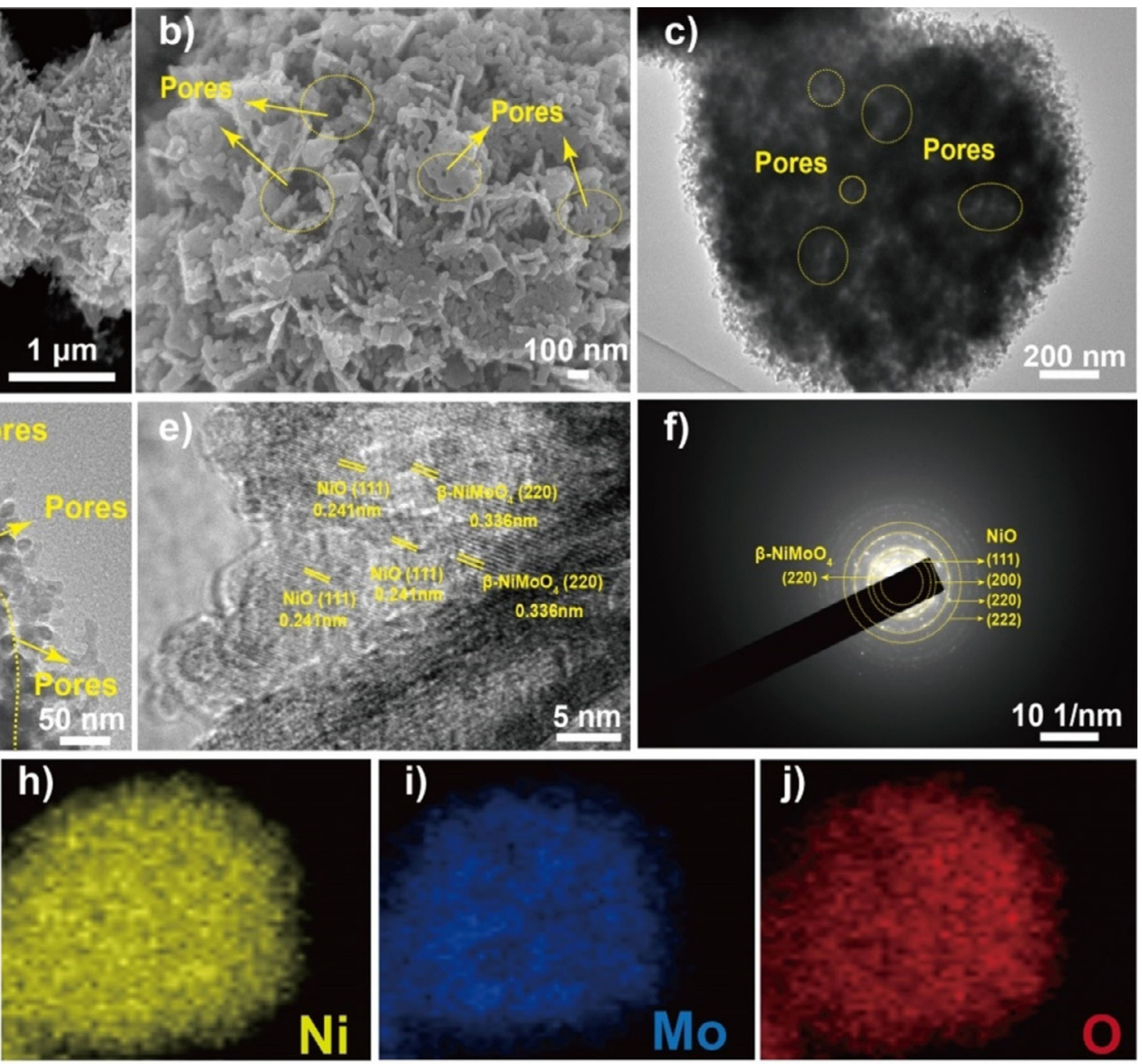

Figure 3. a,b) Representative SEM images at different magnifications of NNMO; c-e) TEM and HRTEM images of as-prepared NNMO; f) SAED pattern; and gj) corresponding EDS elemental mapping images of the NNMO material. 
the volume expansion and effectively maintain the structural integrity and stability. In the selected area electron diffraction (SAED) pattern of the NNMO (Figure $3 \mathrm{f}$ ), the diffraction rings can be assigned to the (111), (200), (220), and (222) planes of the $\mathrm{NiO}$ phase, as well as to the (220) planes of $\beta-\mathrm{NiMoO}_{4}$, respectively, which also indicates the coexistence of the two phases in the NNMO composite. This novel architecture and its composition were further researched by the corresponding energy dispersive $\mathrm{X}$-ray spectroscopy (EDX) elemental mapping images (Figure $3 \mathrm{~g}-\mathrm{j}$ ), which confirm the presence of $\mathrm{Ni}, \mathrm{Mo}$, and $\mathrm{O}$ elements in the NNMO. Moreover, the Ni, Mo, and $\mathrm{O}$ elements are distributed homogeneously in the entire microsphere, indicating the intimate contact between the two components and the formation of heterostructures. On the contrary, the SEM images of the NO (Figure S2a,b in the Supporting Information) show networks constructed from many disor- dered and aggregated rods. The length of the rods is between one and tens of micrometers. Figure $\mathrm{S} 2 \mathrm{c}, \mathrm{d}$ further reveals the general microsphere architecture of $\alpha$-phase $\mathrm{NiMoO}_{4}$ with average diameters of several micrometers. Although the morphologies of $\alpha$-phase and $\beta$-phase $\mathrm{NiMoO}_{4}$ are quite similar, without the introduced $\mathrm{NiO}$ phase, the nanosheets of the NMO microsphere aggregate severely, resulting in fewer pores and inferior structural stability.

For further investigation of the electrochemical behavior of the synthesized materials for Li-ion batteries, cyclic voltammetry (CV) measurements were conducted. Figure 4 a shows the first five consecutive CV curves of the NNMO electrode recorded at a scan rate of $0.1 \mathrm{mV} \mathrm{s}^{-1}$ in the potential range $0.01-$ $3.00 \mathrm{~V}$ versus $\mathrm{Li} / \mathrm{Li}^{+}$. It is evident that from the second to the fifth cycles, these CV profiles overlap well, implying good reversibility and cycling stability of the NNMO electrode during
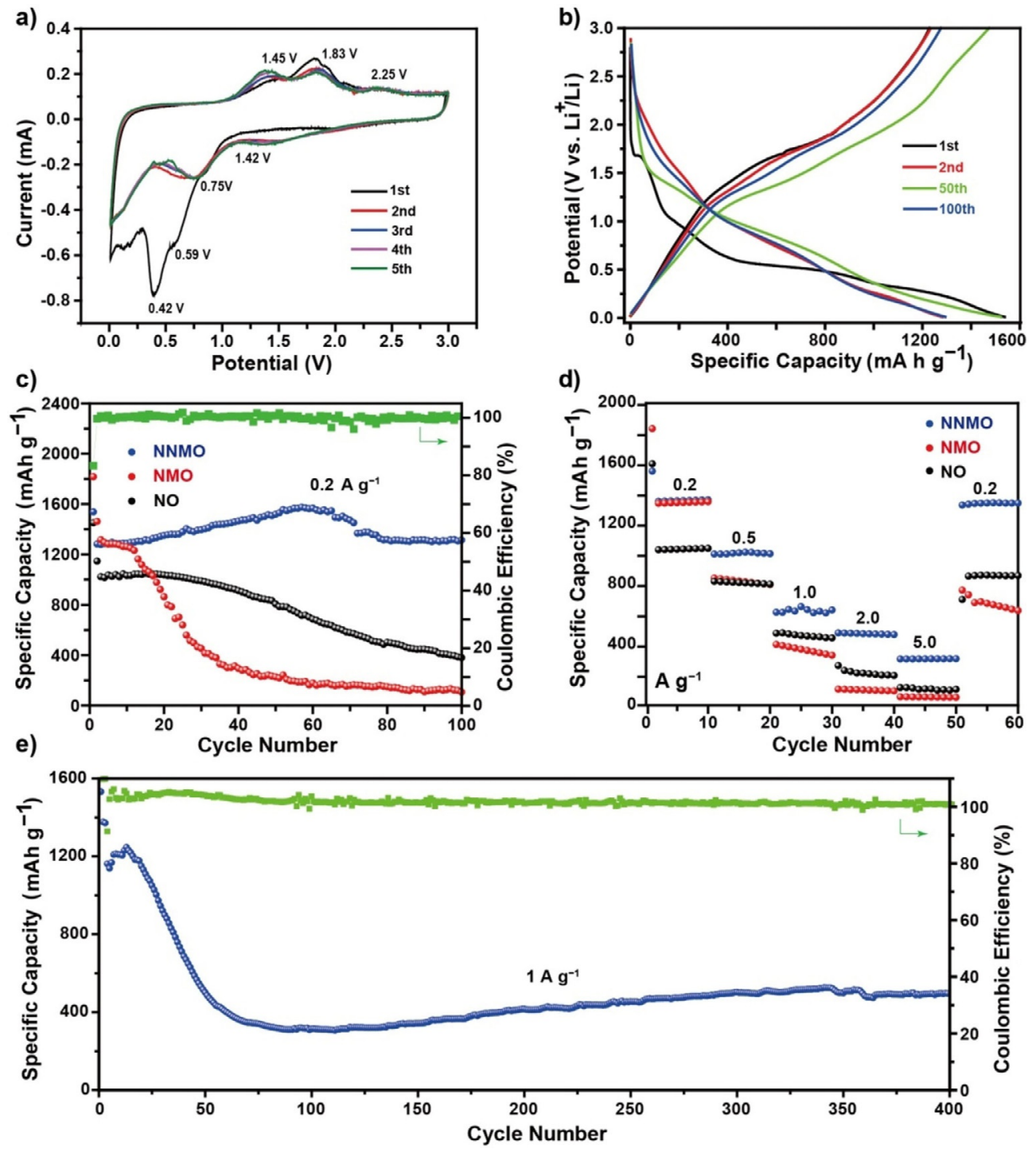

Figure 4. Electrochemical evaluation of NNMO, showing: a) cyclic voltammograms for the first five cycles from 0.01 to $3.00 \mathrm{~V}$; b) galvanostatic charge/discharge voltage profiles versus capacity of NNMO electrode for selected cycles at a current rate of $0.2 \mathrm{Ag}^{-1}$; c) cycling performances of the obtained $\mathrm{NO}$, NMO, and NNMO electrodes at $0.2 \mathrm{Ag}^{-1}$ and Coulombic efficiency of the NNMO electrode at $0.2 \mathrm{Ag}^{-1}$; d) rate performances of the three electrodes at varying current rates of $0.2,0.5,1,2,5$, and $0.2 \mathrm{Ag}^{-1}$; and e) long-term cycling performance at $1 \mathrm{Ag}^{-1}$ of the obtained NNMO electrode and the corresponding Coulombic efficiency. 
electrochemical reactions. In the first cycle, two intense reduction peaks in the cathodic scan occur at around 0.59 and $0.42 \mathrm{~V}$, corresponding to the initial reduction of $\beta-\mathrm{NiMoO}_{4}$ to $\mathrm{Ni}$ and $\mathrm{Mo}\left(\mathrm{NiMoO}_{4}+8 \mathrm{Li}^{+}+8 \mathrm{e}^{-} \rightarrow \mathrm{Ni}+\mathrm{Mo}+4 \mathrm{Li}_{2} \mathrm{O}\right)$ and $\mathrm{NiO}$ to $\mathrm{Ni}$ $\left(\mathrm{NiO}+2 \mathrm{Li}^{+}+2 \mathrm{e}^{-} \rightarrow \mathrm{Ni}+\mathrm{Li}_{2} \mathrm{O}\right)$, combined with the formation of amorphous $\mathrm{Li}_{2} \mathrm{O}$ and SEI layers. ${ }^{[16,36,37]}$ Meanwhile, the broad peaks in the voltage range from 1.4 to $1.8 \mathrm{~V}$ can be ascribed to the oxidation process of $\mathrm{Mo}$ to $\mathrm{Mo}^{6+}\left(\mathrm{Mo}+3 \mathrm{Li}_{2} \mathrm{O} \rightarrow \mathrm{MoO}_{3}+\right.$ $\left.6 \mathrm{Li}^{+}+6 \mathrm{e}^{-}\right)_{1}^{[38]}$ whereas the oxidation of metallic $\mathrm{Ni}$ to $\mathrm{NiO}_{x}$ contributes to the anodic peak located at approximately $1.83 \mathrm{~V}^{[39]}$ In subsequent cycles, the different reduction/oxidation peak pairs of $0.75 / 1.45 \mathrm{~V}$ and $1.42 / 1.79 \mathrm{~V}$ are reversible because of the $\mathrm{Li}$ insertion/extraction process with $\mathrm{MoO}_{3}{ }^{[39,40]} \mathrm{No}$ tably, an anodic peak near $2.25 \mathrm{~V}$ is observed in the NNMO electrode, owing to the conversion reaction of $\mathrm{Ni}$ to $\mathrm{NiO}(\mathrm{Ni}+$ $\left.\mathrm{Li}_{2} \mathrm{O} \rightarrow \mathrm{NiO}+2 \mathrm{Li}^{+}+2 \mathrm{e}^{-}\right) .^{[36]}$ On the other hand, the CV curves of $\mathrm{NO}$ and $\mathrm{NMO}$ electrodes show different behavior from that of the NNMO electrode. In the first cathodic process of the NO electrode, two sharp peaks are observed clearly at 0.437 and $0.398 \mathrm{~V}$, respectively, attributed to the reduction reactions of $\mathrm{NiO}$ and the formation of amorphous $\mathrm{Li}_{2} \mathrm{O}$, as well as a partially irreversible SEl layer. The oxidation of metallic $\mathrm{Ni}$ to $\mathrm{NiO}$ contributes to the anodic peak located at approximately $2.26 \mathrm{~V}$, whereas the anodic peaks located at about $1.805 \mathrm{~V}$ are attributed to the formation of Mo (Figure S3 in the Supporting Information).

Figure $4 \mathrm{~b}$ shows the typical galvanostatic discharge/charge voltage profiles of the NNMO anode from 0.01 to $3.0 \mathrm{~V}$ at a current density of $0.2 \mathrm{Ag}^{-1}$. In the first cycle, the NNMO electrode delivered an initial discharge capacity of $1538 \mathrm{~mA} \mathrm{~h}^{-1}$ and charge capacity of $1282 \mathrm{~mA} \mathrm{hg}^{-1}$, corresponding to an initial Coulombic efficiency (ICE) of $83 \%$, which is higher than those of the NO (80\%) and NMO electrodes (78\%) (Figure S4 in the Supporting Information), demonstrating the high reversibility and good stability of the NNMO electrode during the electrochemical reactions. The higher reversible capacity of the NNMO can be explained by the further delithiation reaction observed above $2.0 \mathrm{~V}$ (Figure $4 \mathrm{a}$ ), which is attributed to the catalytic behavior of the metallic nickel nanocrystals and its unique porous hierarchical structure that can shorten ion diffusion pathways. ${ }^{[41]}$ Furthermore, the NNMO anode exhibited excellent cycling stability. As shown in Figure 4c, the NNMO electrode delivered an initial reversible capacity of $1282 \mathrm{~mA} \mathrm{hg}^{-1}$, which then increased gradually to $1314 \mathrm{~mA} \mathrm{hg}^{-1}$, with a Coulombic efficiency of nearly $100 \%$ after 100 cycles. The feature of increased capacity after the initial cycle is not surprising for a metal-oxide-based material, as it is always ascribed to the reversible growth of polymeric gel-like layers and capacitive interface storage including electric double layer capacitance, as well as an activation process in the nanostructures. ${ }^{[37]}$ On the contrary, rapid capacity fading and inferior capacity retention are clearly observed in the NO and NMO materials. The high electrochemical reversibility and good cycling stability of the NNMO material may be attributed to the following factors: 1) the $\mathrm{Ni}$ nanocrystals generated in situ from the $\mathrm{NiO}$ phase could function as a direct catalyst for the reversible decomposition of intermediate $\mathrm{Li}_{2} \mathrm{O}$ and the oxidation of metallic $\mathrm{Ni}$ and
Mo from $\beta$-NiMoO${ }_{4} ; 2$ ) the $\mathrm{NiO}$ nanosheets can act as a mechanical buffer to stabilize the $\mathrm{NiMoO}_{4}$ phase effectively, thus reducing the self-agglomeration of $\mathrm{NiMoO}_{4}$ nanosheets and maintaining good structural stability; 3 ) the unique hierarchical porous architecture constructed from the small nanosheets will provide more active sites for $\mathrm{Li}^{+}$storage, short ion diffusion pathways, and abundant additional space to release the internal stress induced by volume changes during the discharge/ charge process.

Additionally, the NNMO electrode also exhibited a remarkable rate capability at different current densities. As seen from Figure $4 d$, the NNMO electrode is capable of delivering specific capacities of $1360,1003,631,469$, and $308 \mathrm{~mA} \mathrm{~h} \mathrm{~g}^{-1}$ at current densities of $0.2,0.5,1.0,3.0$, and $5.0 \mathrm{Ag}^{-1}$, respectively. More importantly, the capacity can recover to the initial value (about $1339 \mathrm{mAhg}^{-1}$ ) upon returning the current rate back to $0.2 \mathrm{Ag}^{-1}$ after 60 cycles, indicating the excellent rate capability and good reversibility of the NNMO electrode. On the contrary, both the NO and the NMO electrodes show far inferior capacity retention and severe capacity decay between current densities of 1 and $5 \mathrm{Ag}^{-1}$. The excellent rate capability can be attributed to the synergistic effects of the binary composition and the short lithium diffusion distance in the porous hierarchical NNMO heterostructure. The long cycling performance is also an important parameter for batteries in practical applications. Hence, we further examined the long-term cycling property of the NNMO electrode at a high current density of $1 \mathrm{Ag}^{-1}$ (Figure $4 \mathrm{e}$ ). After 400 cycles, the NNMO anode still retained a high reversible capacity of around $500 \mathrm{mAhg}^{-1}$, and the average Coulombic efficiency was close to $100 \%$. More importantly, the NNMO electrode can maintain its hierarchical porous structure after long-term cycling, demonstrating its good structural stability. The superior structural characteristics, such as the 2D building blocks, large surface area, and abundant pores enable its good structural integrity (Figure S5 in the Supporting Information).

To reveal the boosted charge-transfer behavior in the NNMO heterostructure, we also investigated the kinetic differences of lithium ion diffusion in these anodes. As shown in Figure $5 \mathrm{a}, \mathrm{b}$, the NNMO sample exhibits larger peak intensities and smaller polarization at all scan rates, indicating the greatly enhanced electrochemical kinetics in the NNMO electrode, which are favorable for achieving better rate capability. Furthermore, the significantly promoted charge-transfer process in the NNMO heterostructure can also be proved by the higher diffusion coefficient of $\mathrm{Li}^{+}$compared with that of the NMO sample (Figure $5 \mathrm{c}$ ). Meanwhile, EIS measurements were conducted to research the kinetic differences between various electrodes (Figure S6 in the Supporting Information). Note that the diameter of the semicircle in the high-to-medium-frequency region for the NNMO electrode is much smaller than those for the NMO and NO electrodes, demonstrating the highly improved electron/ion transport dynamic in the heterostructure of the NNMO sample. ${ }^{[42,43]}$ 
a)

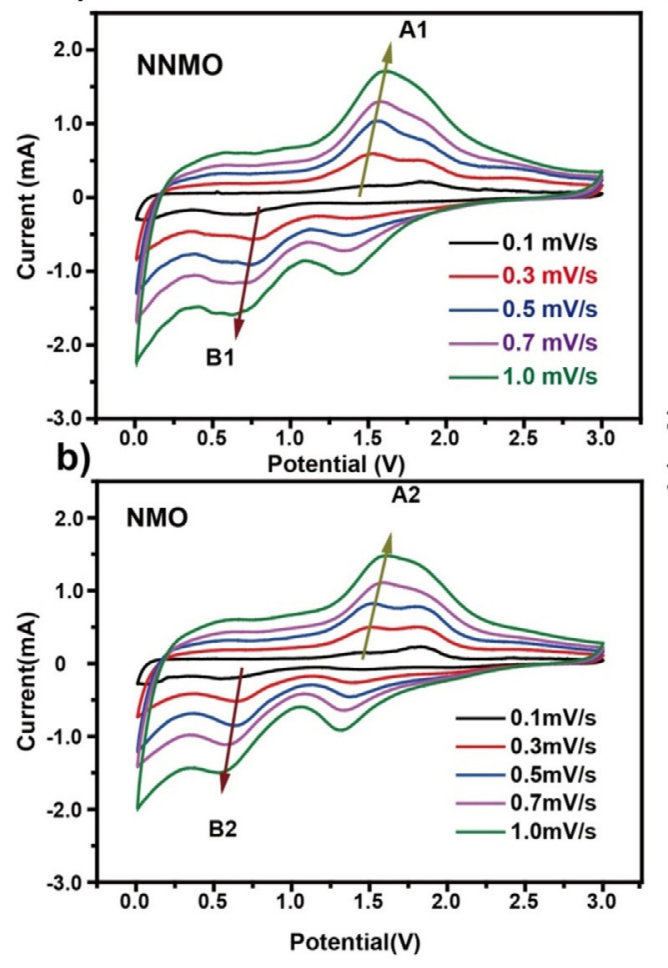

c)

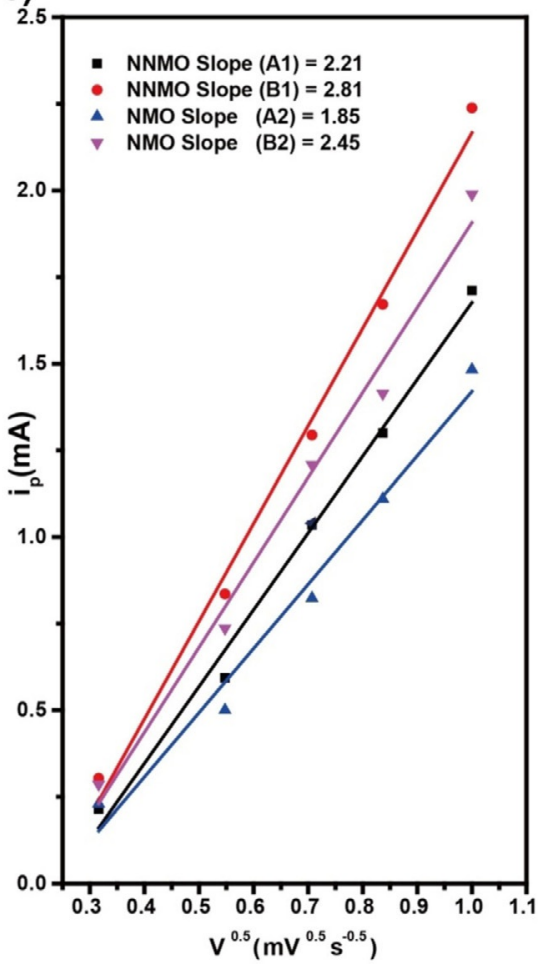

Figure 5. CV curves at different scan rates for LIB cells with a) NNMO and b) NMO materials, and c) the linear relationship between the peak current ( $i_{\mathrm{p}}$ ) and the square root of the scan rate $\left(\tilde{v}^{1 / 2}\right)$ for NNMO electrode.

\section{Conclusion}

In summary, we have developed a facile strategy for the synthesis of a novel hierarchical porous $\mathrm{NiO} / \beta-\mathrm{NiMoO}_{4}$ heterostructure constructed from small nanosheets, featuring a large surface area, short ion-diffusion paths, and good structural stability. By means of synergistically active ternary metal oxides, boosted charge-transfer dynamics, and enhanced electrochemical reversibility, the NNMO heterostructure achieved a high reversible capacity, good rate capability, and superior long-term cyclability for Li-ion batteries. Our material design strategy presented here could be a promising approach for the design of novel heterostructure nanomaterials for high-performance rechargeable batteries.

\section{Experimental Section}

\section{Synthesis of hierarchical porous $\mathrm{NiO} / \boldsymbol{\beta}-\mathrm{NiMoO}_{4}$ heterostruc-} ture

In a typical synthesis process, nickel acetate tetrahydrate $(2 \mathrm{mmol}$, $0.4978 \mathrm{~g})$ and sodium molybdate dehydrate $(1 \mathrm{mmol}, 0.3629 \mathrm{~g})$ with a Ni/Mo molar ratio of 2:1 were slowly added into deionized water $(30 \mathrm{~mL})$ under magnetic stirring for $30 \mathrm{~min}$ to form a transparent solution. The resultant solution was then transferred to a $50 \mathrm{~mL}$ Teflon-lined autoclave, and was kept in an oven at $180^{\circ} \mathrm{C}$ for $12 \mathrm{~h}$. After the hydrothermal reaction, the obtained green product was separated by centrifugation and washed several times with deionized water and ethanol. The resultant composite was then dried at $80^{\circ} \mathrm{C}$ overnight, followed by heating in a tube furnace under an oxygen atmosphere at $550^{\circ} \mathrm{C}$ for $2 \mathrm{~h}$ with a ramp rate of $2{ }^{\circ} \mathrm{Cmin}^{-1}$, yielding the hierarchical NNMO electrode with a Ni/Mo ratio of $2.3: 1$, as determined by inductively coupled plasma (ICP) (Table S1 in the Supporting Information).

\section{Synthesis of single-phase NiO (NO) material}

The synthesis process was similar to that for NNMO. First, nickel chloride hexahydrate $(1.2 \mathrm{mmol}, 0.2852 \mathrm{~g})$, urea $(3 \mathrm{mmol}, 0.1802 \mathrm{~g})$, and cetyltrimethyl ammonium bromide (CTAB, $0.33 \mathrm{mmol}, 0.12 \mathrm{~g}$ ) were added into deionized water $(30 \mathrm{~mL})$ under magnetic stirring for $30 \mathrm{~min}$ to form a transparent solution. Then, the above solution was transferred to a $50 \mathrm{~mL}$ Teflon-lined autoclave and kept in an oven at $180^{\circ} \mathrm{C}$ for $12 \mathrm{~h}$. The obtained slurry was centrifuged, washed with deionized water, and then dried at $80^{\circ} \mathrm{C}$ for $24 \mathrm{~h}$. After thermal oxidation at $550^{\circ} \mathrm{C}$ for $2 \mathrm{~h}$ with a ramp rate of $2{ }^{\circ} \mathrm{Cmin}^{-1}$, the single-phase $\mathrm{NiO}$ material was obtained.

\section{Synthesis of single-phase $\alpha-\mathrm{NiMoO}_{4}$ (NMO) material}

In a typical procedure, nickel chloride hexahydrate $(1.3 \mathrm{mmol}$, $0.3090 \mathrm{~g})$ and sodium molybdate dehydrate $(1.3 \mathrm{mmol}, 0.3527 \mathrm{~g})$ with a Ni/Mo molar ratio of 1:1 were added into a given amount $(30 \mathrm{~mL})$ of distilled water. The resulting solution was then transferred into a Teflon-lined stainless-steel autoclave and maintained at $180^{\circ} \mathrm{C}$ for $12 \mathrm{~h}$. After cooling to room temperature, the slurry was washed several times with deionized water and dried at $80^{\circ} \mathrm{C}$ for $24 \mathrm{~h}$ in an oven. Finally, the dried material, which had a green color, was thermally treated at $550^{\circ} \mathrm{C}$ for $2 \mathrm{~h}$ in a tube furnace under an oxygen atmosphere to yield the $\alpha-\mathrm{NiMoO}_{4}$. 


\section{Material characterization}

The crystalline structure of the materials was investigated by $X$-ray diffraction (XRD, Bruker AXS, D8 diffractometer) with $\mathrm{CuK}_{\alpha}$ radiation at a scanning rate of $1^{\circ} \mathrm{min}^{-1}$. The morphology and energy dispersive spectroscopy (EDS) mapping of the as-prepared products were observed by scanning electron microscopy (SEM; JEOL JSM, $6510 \mathrm{~V}$ ), and the details of the crystal structure were further examined by transmission electron microscopy (TEM; FEI TF20). X-ray photoelectron spectroscopy (XPS) was then conducted on an ESCALAB 250XI photoelectron spectrometer using monochromatic $\mathrm{AlK}_{\alpha}$ radiation under vacuum at $2 \times 10^{-6} \mathrm{~Pa}$. All of the binding energies are referenced to the $\mathrm{C} 1 \mathrm{~s}$ peak at $284.8 \mathrm{eV}$ of the surface adventitious carbon. The actual contents of $\mathrm{Ni}$ and Mo elements were measured by inductively coupled plasma (ICP) emission spectroscopy using a PerkinElmer Optimal 8000 instrument (detection limit: $0.01 \mathrm{ppm}$ for Ni, $0.01 \mathrm{ppm}$ for Mo). Specific surface areas and pore-size distributions were also obtained from $\mathrm{N}_{2}$ adsorption/desorption isotherms recorded at $77 \mathrm{~K}$ with a Micromeritics ASAP 2020 porosimeter. Prior to surface area measurements, the samples were dried under vacuum at $393 \mathrm{~K}$ for $8 \mathrm{~h}$. The surface area was computed using the Brunauer-Emmett-Teller (BET) method, and the pore-size distribution was calculated in accordance with the Barrett-Joyner-Halenda (BJH) method.

\section{Electrochemical measurements}

Electrochemical tests were conducted on CR2025 coin-type cells, which were assembled in an Ar-filled glovebox. The working electrodes were prepared by mixing the active materials, Super $\mathrm{P}$, and Na-carboxymethyl cellulose (CMC) in the weight ratio 60:30:10. The mixture was then pasted on $\mathrm{Cu}$ foil, dried at $80^{\circ} \mathrm{C}$ for $12 \mathrm{~h}$ in a vacuum oven, and pressed at $300 \mathrm{~kg} \mathrm{~cm}^{-2}$. The mass loading of the materials on individual electrodes was approximately $1.15 \mathrm{mg} \mathrm{cm}^{-2}$. Electrochemical measurements were performed using two-electrode coin cells with Li metal as the counter and reference electrode and Celgard (product 2300) film as the separator. The electrolyte consisted of a solution of $1 \mathrm{M} \mathrm{LiPF} 6$ in ethylene carbonate/ diethyl carbonate/ethyl methyl carbonate $(1: 1: 1, v / v / v)$. Electrochemical impedance spectroscopy and cyclic voltammetry were conducted on a $\mathrm{CHI} 660 \mathrm{E}$ electrochemical workstation at different scan rates. The cells were galvanostatically charged/discharged in the voltage range $0.01-3.00 \mathrm{~V}$ versus $\mathrm{Li} / \mathrm{Li}^{+}$at different current densities on a Land CT2001A battery tester.

The Li-ion diffusion coefficient $\left(D_{\mathrm{Li}+}\right)$ was calculated on the basis of the Randles-Sevcik Equation (described previously ${ }^{[3]}$ ), as given in Equation (1), in which $i_{p}$ is the peak current (A), $n$ is the number of transferred electrons, $D_{\mathrm{Li}+}$ is the diffusion coefficient of $\mathrm{Li}$ ions $\left(\mathrm{cm}^{2} \mathrm{~s}^{-1}\right), S$ is the electrode surface area $\left(S=70.85 \mathrm{~cm}^{2}\right), C$ is the concentration of $\mathrm{Li}^{+}$in the electrolyte, and $\tilde{v}$ is the potential scan rate $\left(\mathrm{Vs}^{-1}\right)$.

$i_{p}=\left(2.69 \times 10^{5}\right) n^{3 / 2} S D^{1 / 2} C v^{1 / 2}$

As the electrodes were prepared and tested by the same procedure, the Randles-Sevcik equation could be simplified further, as given in Equation (2), in which $A$ is supposed to be a constant and $A D^{1 / 2}$ is defined as the apparent diffusion coefficient of $\mathrm{Li}^{+}$in the coin cells, which can be calculated by fitting the linear curves.

$i_{p}=A D^{1 / 2} v^{1 / 2}$

\section{Acknowledgements}

Financial support provided by the grant from the National Natural Science Foundation of China (No. 21476063) and the Hubei Provincial Natural Science Foundation of China (2018CFB237) is gratefully acknowledged. We also thank Dr. T. Silver for critical reading of the manuscript.

\section{Conflict of interest}

The authors declare no conflict of interest.

Keywords: electrochemistry . heterostructures . Li-ion batteries $\cdot$ nanostructures $\cdot$ ternary transition metal oxides

[1] J. Mao, T. Zhou, Y. Zheng, H. Gao, H. Liu, Z. Guo, J. Mater. Chem. A 2018, 6, 3284-3303.

[2] T. Zhou, Y. Zheng, H. Gao, S. Min, S. Li, H. Liu, Z. Guo, Adv. Sci. 2015, 2, 1500027.

[3] J. Yang, Y. Wang, W. Li, L. Wang, Y. Fan, W. Jiang, W. Luo, Y. Wang, B. Kong, C. Selomulya, H. Liu, S. Dou, D. Zhao, Adv. Mater. 2017, 29, 1700523.

[4] Y. Liu, T. Zhou, Y. Zheng, Z. He, C. Xiao, W. Pang, W. Tong, Y. Zou, B. Pan, Z. Guo, Y. Xie, ACS Nano 2017, 11, 8519-8526.

[5] Y. Zheng, T. Zhou, X. Zhao, W. Pang, H. Gao, S. Li, Z. Zhou, H. Liu, Z. Guo, Adv. Mater. 2017, 29, 1700396.

[6] G. Zhang, X. Lou, Angew. Chem. Int. Ed. 2014, 53, 9041-9044; Angew. Chem. 2014, 126, 9187-9190.

[7] C. Tan, Z. Lai, H. Zhang, Adv. Mater. 2017, 29, 1701392.

[8] C. Yuan, H. Wu, Y. Xie, X. Lou, Angew. Chem. Int. Ed. 2014, 53, $1488-$ 1504; Angew. Chem. 2014, 126, 1512-1530.

[9] Y. Zhao, X. Li, B. Yan, D. Xiong, D. Li, S. Lawes, X. Sun, Adv. Energy Mater. 2016, 6, 1502175.

[10] Y. Ma, C. Tai, R. Younesi, T. Gustafsson, J. Lee, K. Edström, Chem. Mater. 2015, 27, 7698-7709.

[11] T. Wei, C. Chen, H. Chien, S. Lu, C. Hu, Adv. Mater. 2010, 22, 347-351.

[12] B. Moreno, E. Chinarro, M. Colomer, J. Jurado, J. Phys. Chem. C 2010, $114,4251-4257$.

[13] S. Ratha, A. Samantara, K. Singha, A. Gangan, B. Chakraborty, B. Jena, C. Rout, ACS Appl. Mater. Interfaces 2017, 9, 9640-9653.

[14] D. Cai, D. Wang, B. Liu, Y. Wang, Y. Liu, L. Wang, H. Li, H. Huang, Q. Li, T. Wang, ACS Appl. Mater. Interfaces 2013, 5, 12905-12910.

[15] X. Tian, X. Li, T. Yang, K. Wang, H. Wang, Y. Song, Z. Liu, Q. Guo, Appl. Surf. Sci. 2018, 434, 49-56.

[16] J. Haetge, I. Djerdj, T. Brezesinski, Chem. Commun. 2012, 48, 6726-6728.

[17] M. Cao, Y. Bu, X. Lv, X. Jiang, L. Wang, S. Dai, M. Wang, Y. Shen, Appl. Surf. Sci. 2018, 435, 641-648.

[18] Y. Zheng, T. Zhou, C. Zhang, J. Mao, H. Liu, Z. Guo, Angew. Chem. Int. Ed. 2016, 55, 3408-3413; Angew. Chem. 2016, 128, 3469-3474.

[19] E. Pomerantseva, Y. Gogotsi, Nat. Energy 2017, 2, 17089.

[20] L. Hou, L. Lian, L. Zhang, G. Pang, C. Yuan, X. Zhang, Adv. Funct. Mater. 2015, 25, 238- 246.

[21] L.-Q. Mai, F. Yang, Y.-L. Zhao, X. Xu, L. Xu, Y.-Z. Luo, Nat. Commun. 2011, 2, $381-385$.

[22] Z. Sun, W. Ai, J. Liu, X. Qi, Y. Wang, J. Zhu, H. Zhang, T. Yu, Nanoscale 2014, 6, 6563-6568.

[23] Y. Zhang, Q. Zhuo, X. Lv, Y. Ma, J. Zhong, X. Sun, Electrochim. Acta 2015, $178,590-596$.

[24] M. Li, Y. Yin, C. Li, F. Zhang, L. Wan, S. Xu, D. Evans, Chem. Commun. 2012, 48, 410-412.

[25] Y. Chen, Y. Wang, X. Shen, R. Cai, H. Yang, K. Xu, A. Yuan, Z. Ji, J. Mater. Chem. A 2018, 6, 1048-1056.

[26] L. Cong, H. Xie, J. Li, Adv. Energy Mater. 2017, 7, 1601906.

[27] Y. Guo, L. Yu, C. Wang, Z. Lin, X. Lou, Adv. Funct. Mater. 2015, 25, $5184-$ 5189. 
[28] W. Liu, T. Zhou, Y. Zheng, J. Liu, C. Feng, Y. Shen, Y. Huang, Z. Guo, ACS Appl. Mater. Interfaces 2017, 9, 9778-9784.

[29] W. Xiao, J. Chen, C. Li, R. Xu, X. Lou, Chem. Mater. 2010, 22, 746-754.

[30] S. Zhang, F. Yao, L. Yang, F. Zhang, S. Xu, Carbon 2015, 93, 143-150.

[31] A. Vu, Y. Qian, A. Stein, Adv. Energy Mater. 2012, 2, 1056-1085.

[32] K. Xiao, L. Xia, G. Liu, S. Wang, L. Ding, H. Wang, J. Mater. Chem. A 2015, 3, 6128-6135.

[33] B. Wang, S. Li, X. Wu, J. Liu, W. Tian, Phys. Chem. Chem. Phys. 2016, 18, $908-915$.

[34] J. Yan, Z. Fan, W. Sun, G. Ning, T. Wei, Q. Zhang, R. Zhang, L. Zhi, F. Wei, Adv. Funct. Mater. 2012, 22, 2632-2641.

[35] B. Wang, S. Li, X. Wu, W. Tian, J. Liu, M. Yu, J. Mater. Chem. A 2015, 3, $13691-13698$.

[36] C. Kim, J. Jung, K. Yoon, D. Youn, S. Park, I. Kim, ACS Nano 2016, 10, $11317-11326$

[37] X. Sun, W. Si, X. Liu, J. Deng, L. Xi, L. Liu, C. Yan, O. Schmidt, Nano Energy 2014, 9, 168-171.
[38] X. Zhang, X. Song, S. Gao, Y. Xu, X. Cheng, H. Zhao, L. Huo, J. Mater. Chem. A 2013, 1, 6858-6864.

[39] J. Ahn, G. Park, Y. Kang, J. Lee, Electrochim. Acta 2015, 174, 102-110.

[40] G. Zhao, N. Zhang, K. Sun, J. Mater. Chem. A 2013, 1, $221-224$.

[41] C. Hua, X. Fang, Z. Wang, L. Chen, Chem. Eur. J. 2014, 20, 5487-5491.

[42] K. Cao, L. Jiao, H. Liu, Y. Liu, Y. Wang, Z. Guo, H. Yuan, Adv. Energy Mater. 2015, 5, 1401421.

[43] T. Zhou, W. Pang, C. Zhang, J. Yang, Z. Chen, H. Liu, Z. Guo, ACS Nano 2014, 8, 8323-8333.

Manuscript received: April 30, 2018

Revised manuscript received: June 1, 2018

Accepted manuscript online: June 19, 2018

Version of record online: July 3, 2018 